\title{
"Smell improvement by anti-IgE and anti-IL 5 biologics in patients with CRSwNP and severe asthma- A real life study"
}

\author{
Blanca Barroso ${ }^{1}$, Marcela Valverde ${ }^{1}$, Isam Alobid ${ }^{2}$, Olaguibel $\mathrm{JM}^{2}$, Manuel Rial Prado ${ }^{2}$, \\ Santiago Quirce ${ }^{2}$, Ebymar Arismendi ${ }^{2}$, Pilar Barranco ${ }^{2}$, Diana Betancor ${ }^{1}$, IRINA \\ BOBOLEA ${ }^{2}$, Blanca Cárdaba ${ }^{2}$, Maria-Jesús Cruz $^{2}$, Elena Curto², Javier \\ Domínguez-Ortega ${ }^{2}$, Francisco-Javier González-Barcala ${ }^{2}$, Carlos Martinez Rivera², Ignacio \\ Mahillo $^{1}$, Xavier Muñoz ${ }^{2}$, César Picado ${ }^{2}$, Vicente Plaza ${ }^{2}$, Jose Rodrigo-Muñoz ${ }^{2}$, Lorena \\ Soto Retes ${ }^{2}$, Antonio Valero ${ }^{2}$, VICTORIA DEL POZO ${ }^{2}$, Joaquim Mullol ${ }^{2}$, and Joaquin \\ Sastre $^{1}$ \\ ${ }^{1}$ Hospital Universitario Fundacion Jimenez Diaz \\ ${ }^{2}$ Centro de Investigacion Biomedica en Red
}

November 11, 2021

\begin{abstract}
Background. Chronic rhinosinusitis with nasal polyps (CRSwNP), characterized by partial (hyposmia) or total (anosmia) loss of smell, is commonly associated with asthma and/or nonsteroidal anti-inflammatory drug-exacerbated respiratory disease (NERD), worsens disease severity and quality of life. The objective of this study was to determine whether, in real-life conditions, biological treatments prescribed for severe asthma can improve olfaction in patients with CRSwNP. A further objective was to compare smell improvement in N-ERD and non-N-ERD subgroups. Methods. A multicenter, non-interventional, retrospective, observational study was performed, including 206 patients with severe asthma undergoing biological treatment (omalizumab, mepolizumab, benralizumab, or reslizumab) with CRSwNP. Results. Improved olfaction was found after treatment with all monoclonal antibodies: omalizumab (35.8\%), mepolizumab (35.4\%), reslizumab (35.7\%), and benralizumab (39.1\%), with no differences between groups. Patients with atopy, greater use of short course systemic corticosteroids, and larger polyp size were more likely to experience improvement in smell. The proportion of patients experiencing smell improvement was similar between the N-ERD (37\%) and non-N-ERD (35.7\%) groups. Conclusions. This is the first study to compare real-life improvement in sense of smell among patients undergoing long-term treatment with omalizumab, mepolizumab, reslizumab, or benralizumab for severe asthma and associated CRSwNP. Approximately 4 out of 10 patients reported a subjective improvement in sense of smell (with non-significant differences between biologic drugs). No differences were found in smell improvement between the N-ERD and non-N-ERD group.
\end{abstract}

\section{Hosted file}

Main_text.doc available at https://authorea.com/users/445612/articles/545101--smellimprovement-by-anti-ige-and-anti-il-5-biologics-in-patients-with-crswnp-and-severeasthma-a-real-life-study

\section{Hosted file}

Figures.docx available at https://authorea.com/users/445612/articles/545101--smellimprovement-by-anti-ige-and-anti-il-5-biologics-in-patients-with-crswnp-and-severeasthma-a-real-life-study 